\title{
Theoretical studies of furan and thiophene nanothreads: structures, cycloaddition barriers and activation volumes
}

\author{
Bo Chen, ${ }^{1,2,3}$ Vincent H. Crespi, ${ }^{1,4,5,6}$ Roald Hoffmann, ${ }^{7}$
}

${ }^{1}$ Department of Chemistry, The Pennsylvania State University, University Park PA, 16802, USA

${ }^{2}$ Donostia International Physics Center, Paseo Manuel de Lardizabal, 4, 20018 Donostia-San Sebastian, Spain

${ }^{3}$ IKERBASQUE, Basque Foundation for Science, Plaza Euskadi 5, 48009 Bilbao, Spain

${ }^{4}$ Department of Physics, The Pennsylvania State University, University Park PA, 16802, USA

${ }^{5}$ Materials Research Institute, The Pennsylvania State University, University Park PA, 16802, USA

${ }^{6}$ Department of Materials Science and Engineering, The Pennsylvania State University, University Park PA, 16802, USA

${ }^{7}$ Baker Laboratory, Department of Chemistry and Chemical Biology, Cornell University, Ithaca, New York 14853, USA

\section{bo.chen@dipc.org; rh34@cornell.edu}

\begin{abstract}
In this theoretical study we examine several aspects of the formation, structure, and stability of the most ordered nanothreads yet made, those derived from furan and thiophene. First, we look at the enthalpic consequences and activation barriers of the
\end{abstract}


first two steps of oligomerization by a Diels-Alder mechanism. The ca. $20 \mathrm{GPa}$ difference in the synthetic pressures (furan lower) is explainable in terms of greater loss of aromaticity by the thiophene. Subsequent steps have understandably lower barriers. We show explicitly how pressure affects the reaction profiles, operating through the volume decrease in the transition state and onward to the product molecule. The interesting option of polymerization proceeding in one or two directions opens up the possibility of polymers with two opposing and cumulative dipole moments. The computed activation volumes are consistently more negative for likely initial furan (compared with thiophene) polymerization steps, in accord with the lower onset pressure of furan polymerization. In the second part of our study we examine the energetics of the likely polymers. Three ordered polymer structures compete in enthalpy -- a syn one, with all $\mathrm{O} / \mathrm{S}$ on the same side, an anti one, S/O alternating, and a syn-anti isomer, with segments of four monomers repeating. The syn polymer, if not allowed to distort, is at high enthalpy relative to the other two. The origin of the destabilization is apparent, being S/O lone-pair repulsion, understandably greater for S than $\mathrm{O}$ at the $2.8 / 2.6 \AA$ separation. Set free, the syn isomers curve or arc, in two- or three-dimensional (helical) ways, whose energetics are traced in detail. The syn polymer can also stabilize itself by the thread twisting into zig-zag or helical enthalpic minima. Release of strain in a linear thread as the pressure is relaxed to $1 \mathrm{~atm}$, with consequent thread curving, is a likely mechanism for the observed loss of crystalline order in the polymer as it is returned to ambient pressure.

\section{Introduction}

A defining feature of nanothreads is their unique combination of extreme thinness (only a few $\AA$ in diameter) and rigidity (multiple covalent bonds connecting each unit). This feature distinguishes nanothreads both from traditional polymers, 
which are generally flexible (by rotation around single bonds) and from nanotubes or nanowires, which are normally much thicker. Some ladder polymers ${ }^{1,2}$ share the features of nanothreads. Rigidity is a defining property of hard (not soft) condensed matter; when accompanied by periodicity (for which it is a precondition), it is intimately associated with a manifold of condensed-phase properties. These include transverse phonons, unusual features of the electronic band structure, and numerous property couplings between mechanical and electronic/optical degrees of freedom, particularly when broken symmetry is required. Expressing rigidity at the extreme limits of thinness accentuates any physical property that depends on gradients in properties across the width of an object, such as flexoelectricity. And it enables expression of properties normally quenched in higher-dimensional rigid lattices, such as torsional modes at typical optical-phonon frequencies. More speculatively, the induction of rigidity at high pressure may enable orbital geometries normally accessible only under high compression to be recovered to ambient conditions, supported by the extensional and torsional rigidity of the thread's carbon backbone. Combining physical rigidity with chemical kinetic control one could design nanothread precursors that capture and recover molecular orbital alignments, so long as the ligands in question can be anchored to the thread backbone.

Nanothreads were first synthesized from benzene under pressure in diamond anvil cells or Paris-Edinburgh cells, ${ }^{3,4}$ but the high-pressure solid-state synthesis technique appears to be quite general for unsaturated hydrocarbons. To date, nanothreads have been synthesized from benzene, ${ }^{3,4}$ pyridine, ${ }^{5}$ aniline, ${ }^{6} \mathrm{a}$ naphthalene/octafluoronaphthalene cocrystal, ${ }^{7}$ thiophene, ${ }^{8}$ cubane, ${ }^{9}$ and very recently a phenol/pentafluorophenol cocrystal, ${ }^{10}$ and furan. ${ }^{11}$ It is expected that more polymers of this kind are coming, and that sample sizes of certain threads will rapidly expand as synthesis pressures continue to fall. 
In nanothread synthesis from aromatic molecules, pressure provides a negative $\mathrm{p} \Delta \mathrm{V}$ term of enthalpy ${ }^{12}$ to help overcome the high reaction barriers, typically insurmountable at ambient pressure, due to loss of aromaticity of the precursor molecules in the initiation of $\mathrm{C}-\mathrm{C}$ bond formation. Two types of reaction mechanisms for nanothread formation-concerted [4+2] polymerization and radical-type polymerization-were previously proposed..$^{13}$ The latter appears to be less favored, as initial stages involving the formation of radical intermediates generally have higher intrinsic barriers at $1 \mathrm{~atm}$ and/or a less negative $\mathrm{p} \Delta \mathrm{V}$ term (only one bond forms initially), compared with [4+2] cycloadditions forming two bonds in a concerted manner.

Furan and thiophene, two aromatics similar in structure, were recently shown to form nanothreads at $15 \mathrm{GPa}^{11}$ and $35 \mathrm{GPa},{ }^{8}$ respectively. This $20 \mathrm{GPa}$ difference in synthetic pressure is one thing we will study in this paper. Three characteristic structures of such threads, based on [4+2] cycloaddition pathways, were proposed (Figure 1 shows them in idealized form, not optimized in geometry). In contrast to the complex structural variety of benzene or pyridine nanothreads, as shown in previous enumerations, ${ }^{14,15}$ furan and thiophene nanothreads have less structural variety. This is due to the smaller 5-membered rings, which contain only two double bonds per ring available for polymerization. This lack of variety may be associated with a higher degree of structural order. 

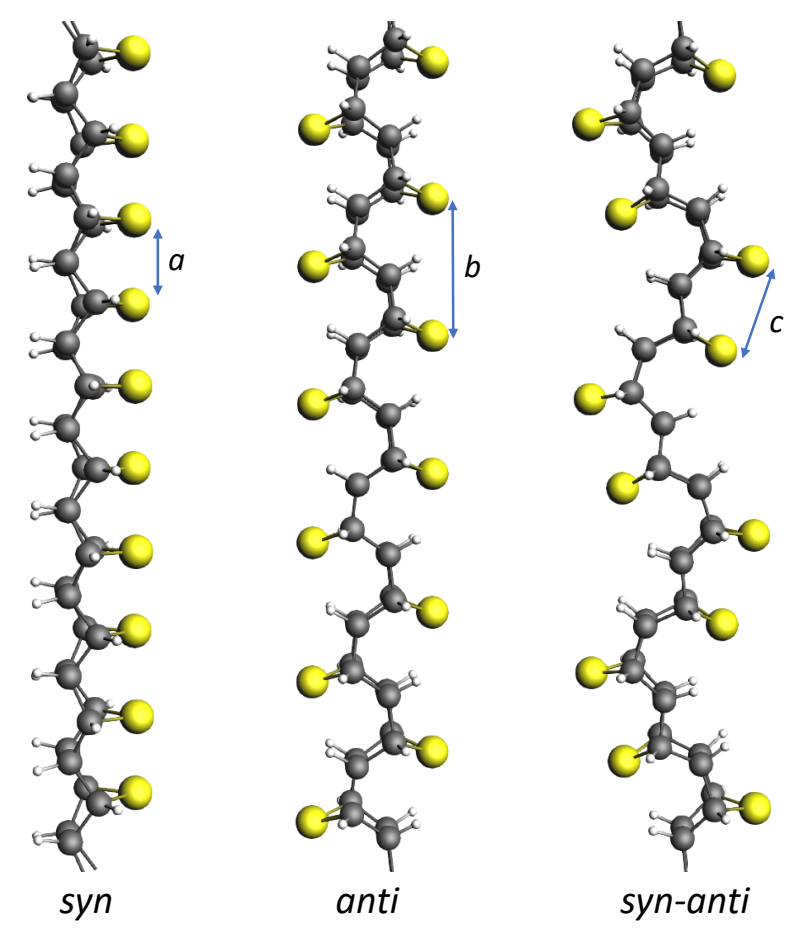

Figure 1. Three characteristic furan or thiophene nanothread structures, in idealized form. Carbons in grey, hydrogens in white, and chalcogens (O or $\mathrm{S}$ ) in yellow. In the DFT-relaxed (using the PBE functional) structures, $a=2.6 \AA$, $b=4.6 \AA$, and $c=2.8 \AA$ for furan threads, and $a=2.8 \AA, b=4.7 \AA$, and $c=3.2 \AA$ for thiophene threads.

Focusing on the relationships of neighboring chalcogens (O or $S$ ) in a thiophene thread structure-whether they are on the same side or opposite sides of the thread-a syn/anti nomenclature naturally comes to mind, and was originally used. ${ }^{8}$ Based on the closeness of the match to experimentally observed d-spacings of the three Friedel pairs of the quasi six-fold diffraction pattern, simulations of packed nanothread crystals suggest that (for both furan and thiophene nanothreads) the anti and/or syn-anti structures, with more elliptical cross sectional thread shapes, are the ones observed. ${ }^{8,11}$ The latest solid-state NMR analyses of a furan thread sample produced in a ParisEdinburgh cell, along with chemical shift calculations (by DFT), identified the fully 
saturated anti structure and some partially saturated polymer structures, while excluding the syn and syn-anti structures. ${ }^{16}$

Energetically, the anti and syn-anti structures are more favorable than the syn structure, due to the absence of the apparent steric hindrance between the closelypositioned chalcogens, an obvious feature of the idealized syn structure. ${ }^{8,11}$ We will give a more detailed analysis of the structures and their energetics in this paper.

If one thinks about how the possible threads form, another nomenclature, now of endo vs exo aggregation, is time-honored for cycloadditions. It is best to approach it through an example. The very first [4+2] cycloaddition offers two possibilities (Figure 2). The linguistic choice, to call the product that puts the two longer ring fragments "on the same side" endo, its counterpart exo, is as arbitrary as it sounds. But we need the distinction between the resulting constitutional isomers.

The number of isomers grows exponentially as the reaction propagates, for each subsequent cycloaddition induces the same endo/exo isomerism. The syn, anti, and synanti nanothread structures in Figure 1 correspond to the [4+2] cycloadditions being all endo, all exo, and alternating endo and exo. The solid-state structures of furan and thiophene, from which nanothreads were made, likely constrain the orientations of individual molecules during polymerization, and thus may limit the structural possibilities. The intrinsic endo/exo selectivity (in a gas-phase reaction) and the packing efficiency of the threads as they form under pressure, both of which are related to the nonbonded $\mathrm{X} \cdots \mathrm{X}(\mathrm{X}=\mathrm{O}$ or $\mathrm{S})$ repulsion, a steric effect, may also influence the structure of the nanothread formed. 

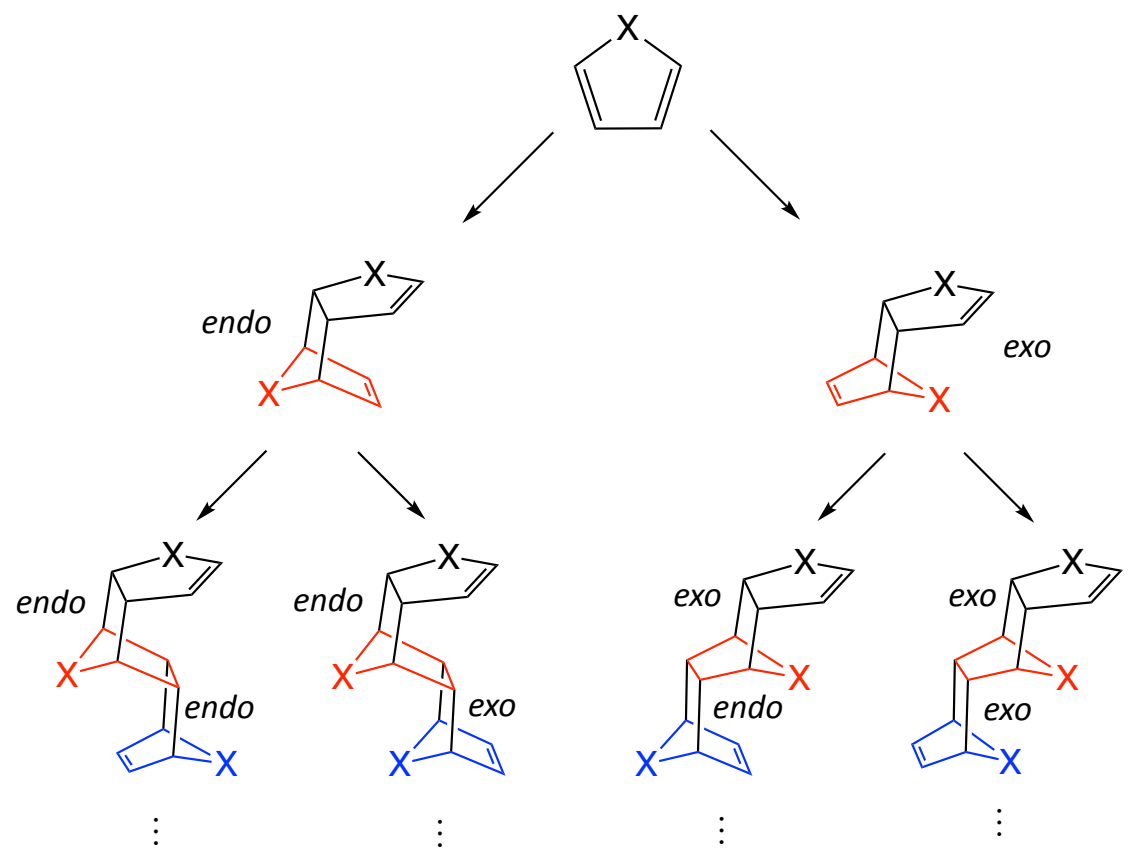

Figure 2. Isomerism of [4+2] cycloadditions in the process of nanothread formation from furan $(X=O)$ or thiophene $(X=S)$. The first ring added to the initial monomer black ring is in read. The second step adds blue rings.

In this paper, we compare barriers and activation volumes of furan and thiophene [4+2] cycloadditions, and the structures of furan/thiophene nanothreads.

\section{[4+2] cycloaddition barriers}

First, we would like to understand the large, $20 \mathrm{GPa}$ difference in the synthetic pressures for thiophene and furan nanothreads. As we will show in the next section, larger (more negative) volumes of activation are calculated for chain-initiating furan $[4+2]$ cycloadditions than thiophene. Therefore, the reduction of enthalpic barrier by pressure should be greater for furan than for thiophene polymerizations. This is one of 
the reasons that furan nanothreads form at a lower pressure than thiophene nanothreads do. Another reason could be that the intrinsic electronic-energy barrier of the thiophene reaction is higher at $1 \mathrm{~atm}$. This is readily understood considering that thiophene is more aromatic, and therefore less susceptible to initial reactions in which that aromaticity is lost, than furan. ${ }^{17,18}$ As shown in Figure 3, the computed [4+2] cycloaddition barriers for thiophene $(41 \mathrm{kcal} / \mathrm{mol}$, after slash on the uppermost arrows) are indeed higher than those for furan (32 kcal/mol, before slash).

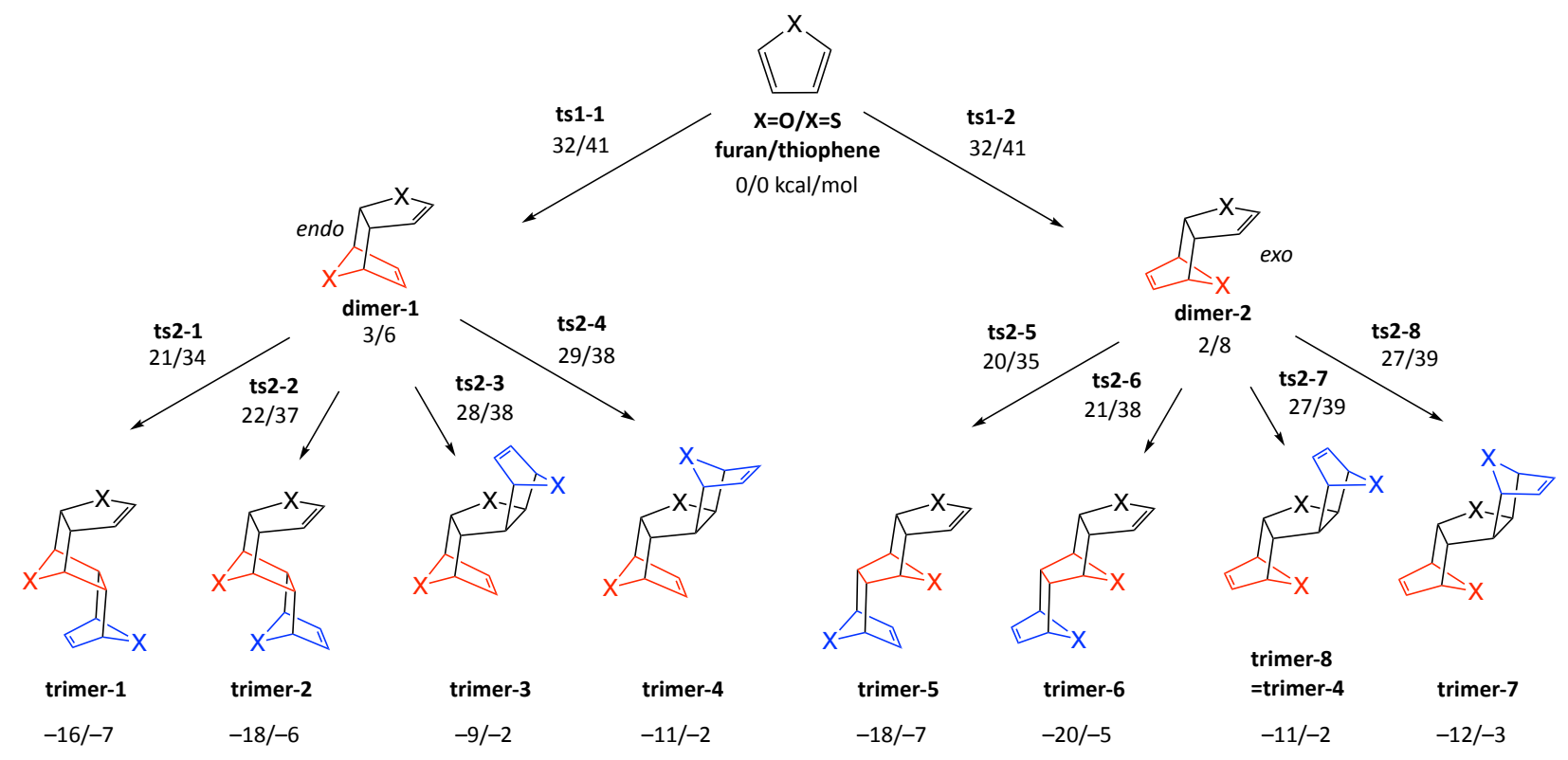

Figure 3. Potential energy profiles (in $\mathrm{kcal} / \mathrm{mol}$ ) at $1 \mathrm{~atm}$ of the first two [4+2] cycloaddition steps in the polymerizations of furan $(X=0) /$ thiophene $(X=S)$, calculated at the DLPNO-CCSD (T)/cc-pVTZ//B3LYP/6-31G(d) level of theory. The numbers adjacent to the arrows are transition state (structures not shown) energies and the numbers adjacent to the structures are the energies of the adducts, all relative to isolated furan/thiophene molecules. Enantiomers (isomers with opposite chirality) are not considered. See Figure S1 in SI for the energetics calculated using other methods. In the stepwise addition sequence, the first rings added are in red, the second ones in blue. 
We should remark at this point that argumentation from loss of aromaticity is only part of the story. In dimerizing $\pi$-electron systems, the conversion of one $\pi$ bond into two $\sigma$ bonds is enthalpically a very much downhill process. Thus, the $\Delta \mathrm{H}$ for three ethylenes to cyclohexane is $-67 \mathrm{kcal} / \mathrm{mol}$. The all-saturated furan and thiophene nanothreads are thermodynamically more stable than the reactants that lead to them. We can observe the trend even for the trimer energies shown, all negative.

The endo and exo pathways in the first [4+2] cycloaddition of furan/thiophene have nearly the same activation barrier. However, there is a slight preference (1-2 $\mathrm{kcal} / \mathrm{mol}$ ) for the exo dimer-2 of furan and for the endo dimer-1 of thiophene.

The dimers formed in the first cycloaddition each have two double bonds, one in the top (black) ring and the other in the bottom (red) ring. Because of the conjugation with a heteroatom $\mathrm{O}$ or $\mathrm{S}$, the double bond in the black ring is more electron-rich than the other double bond. In favorable $4+2$ cycloadditions, the diene and the alkene (dienophile) have opposite polarities in the $\pi$ systems, one being electron-rich and the other electron-deficient. ${ }^{19}$ In contrast, when the diene and alkene are both electronrich, as in the case of cycloaddition between the black double bond and the third, blue furan/thiophene ring (Figure 3), the cycloaddition is unfavorable. Computations indeed give higher barriers for the dimer-to-trimer cycloadditions to the black rings. Correspondingly, the adducts trimer-3 and trimer-4 (or trimer-7 and trimer-8) are less stable than dimer-1 and dimer-2 (or trimer-5 and trimer-6) that are formed by cycloadditions to the red rings.

Despite the understood difference in the preferences between the cycloadditions with the black and the red double bonds of a dimer, both of these dimer-to-trimer reactions were calculated to have lower barriers than the cycloadditions initiating 
polymerization at $1 \mathrm{~atm}$ (Figure 3 ). This can be readily rationalized by noting that the initial cycloaddition breaks the aromaticity of two reactant molecules, while the cycloadditions that follow disrupt the aromaticity of only one molecule.

The initiation calculated to be the rate-limiting step may suggest bidirectional growth of the thread from the dimer, where the thread is extended in both directions including the one involving the relatively unfavorable cycloaddition to the black double bond in the dimer (reaction to trimers-3, $-4,-7$ and -8 , with subsequent chain extension). However, if the kinetic preferences of dimer-to-trimer cycloadditions can be resolved in the polymerization process, e.g., by pressure and crystal structure constraints, then monodirectional growth of the thread is expected and the black double bond in the dimer would never react.

Bidirectional growth of a chain polymer seems rare, ${ }^{20}$ and in this case, would produce a "double-headed" nanothread with a "growth inversion center" (the first black ring) in the middle. If each step in the polymerization builds up a dipole moment in the thread, however small, along the thread direction, then the double-headed growth will produce a thread with two large, opposing dipole moments. Such a structure has not been reported as far as we know, and may have intriguing optoelectronic or electromechanical properties as a rigid, one-dimensional quadrupole.

Focusing on the monodirectional growth pathway, Figure 3 also shows that the energies of corresponding syn and anti structures are within $2 \mathrm{kcal} / \mathrm{mol}$ (e.g., trimer-2 vs. trimer-1, and trimer-6 vs. trimer-5), following the nomenclature in Figure 1. So are the transition states leading to these trimers (e.g., ts2-2 vs. ts2-1). Note that the small energetic difference between the syn and anti structures are for short oligomers in 
the gas-phase; the situation may be quite different for long threads packed in a crystal, especially for thiophene, which suffers more (sterically) from syn configurations.

\section{The effect of pressure on reaction barriers}

Two questions we would like to answer in this section are (1) what is the effect of pressure on the endo/exo selectivity that exists in every step of the $4+2$ polymerization in Figure 3, and (2) would pressure have a similar or different effect on the polymerization of furan compared with thiophene. In order to answer these questions, we calculated the reaction profiles at different pressures and the activation volumes of 5 reaction steps in Figure 3; they are furan/thiophene $\rightarrow$ dimer-1 via ts 11 (reaction A), furan/thiophene $\rightarrow$ dimer-2 via ts1-2 (reaction B), dimer-1 $\rightarrow$ trimer-1 via ts2-1 (reaction $\mathrm{C}$ ), dimer-1 $\rightarrow$ trimer-2 via ts2-2 (reaction $\mathrm{D}$ ), dimer-1 $\rightarrow$ trimer-4 via ts2-4 (reaction $\mathrm{E}$ ). The comparisons of reactions $\mathrm{A}$ and $\mathrm{B}$, and $\mathrm{C}$ and D, are designed to gauge the effects of pressure on the endo/exo selectivities, respectively, in the initiation step and in the chain lengthening step. It is assumed that the effect of pressure on the chain lengthening steps is the same no matter whether the chain grows from dimer-1 or dimer-2, so that the reactions of the right-hand branch in Figure 3 may be ignored. Reaction $\mathbf{E}$ is considered because it is a chain lengthening step in a different direction from those in reactions $\mathbf{C}$ and $\mathbf{D}$.

The set of reactions A-E should be a good representation for all the reactions one may encounter in the $4+2$ polymerization of furan/thiophene. The system is complicated; to keep our wits about us, we duplicate part of Figure 3, and mark on it the reactions $\mathbf{A}$ to $\mathbf{E}$ studied (Scheme 1). 
Scheme 1 . The five reactions studied by high-pressure calculations.

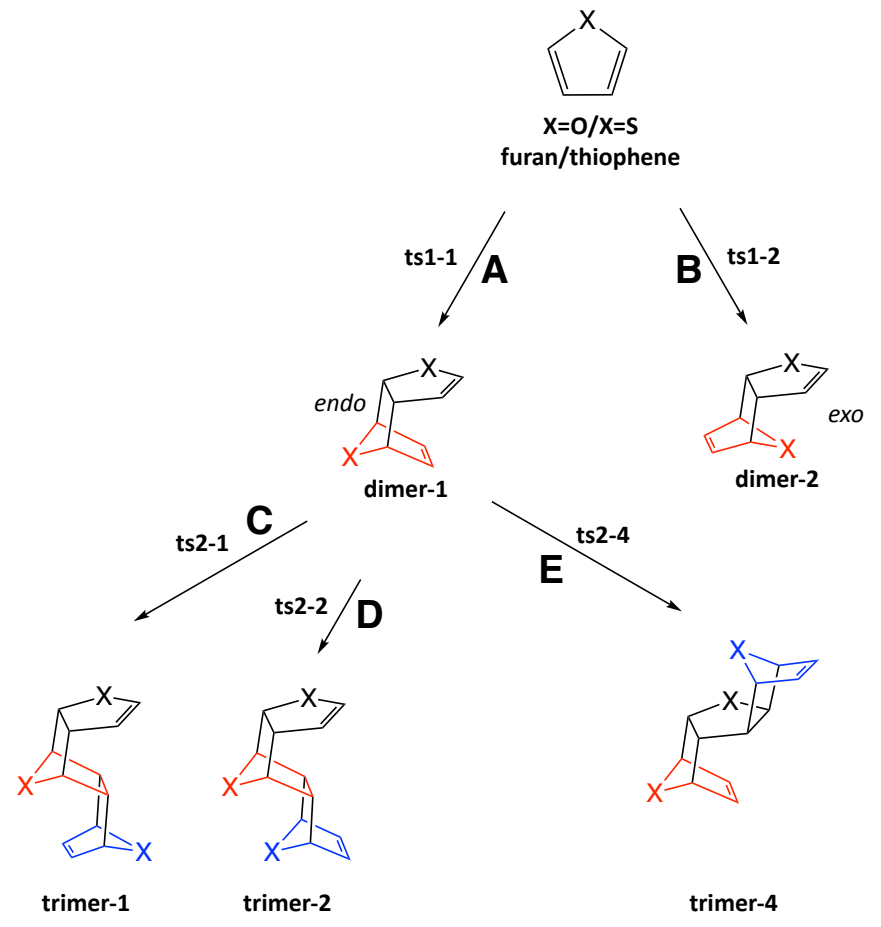

Figure 4 shows the profiles of reactions A-E for furan at different pressures. The profiles for thiophene reactions are very similar to those for furan reactions and are given in Figure S2 in SI. In fact, the profiles in Figure 4 are also very similar in appearance to each other, with late transition states (a transition state that is closer to the product than to the reactant along the reaction coordinate) and decreasing reaction barriers as the pressure increases; these profiles mainly differ by the calculated values of the enthalpic barrier heights and product enthalpies. All these reactions have a similar, monotonically decreasing volume profile. For this reason, only the volume profile for reaction A is shown in Figure 4. The obvious implication is that pressure will facilitate these uniformly volume-decreasing transformations. Entropy changes 
must not be forgotten, but this is where confinement in a crystal lattice is a great advantage over reaction in fluid media.
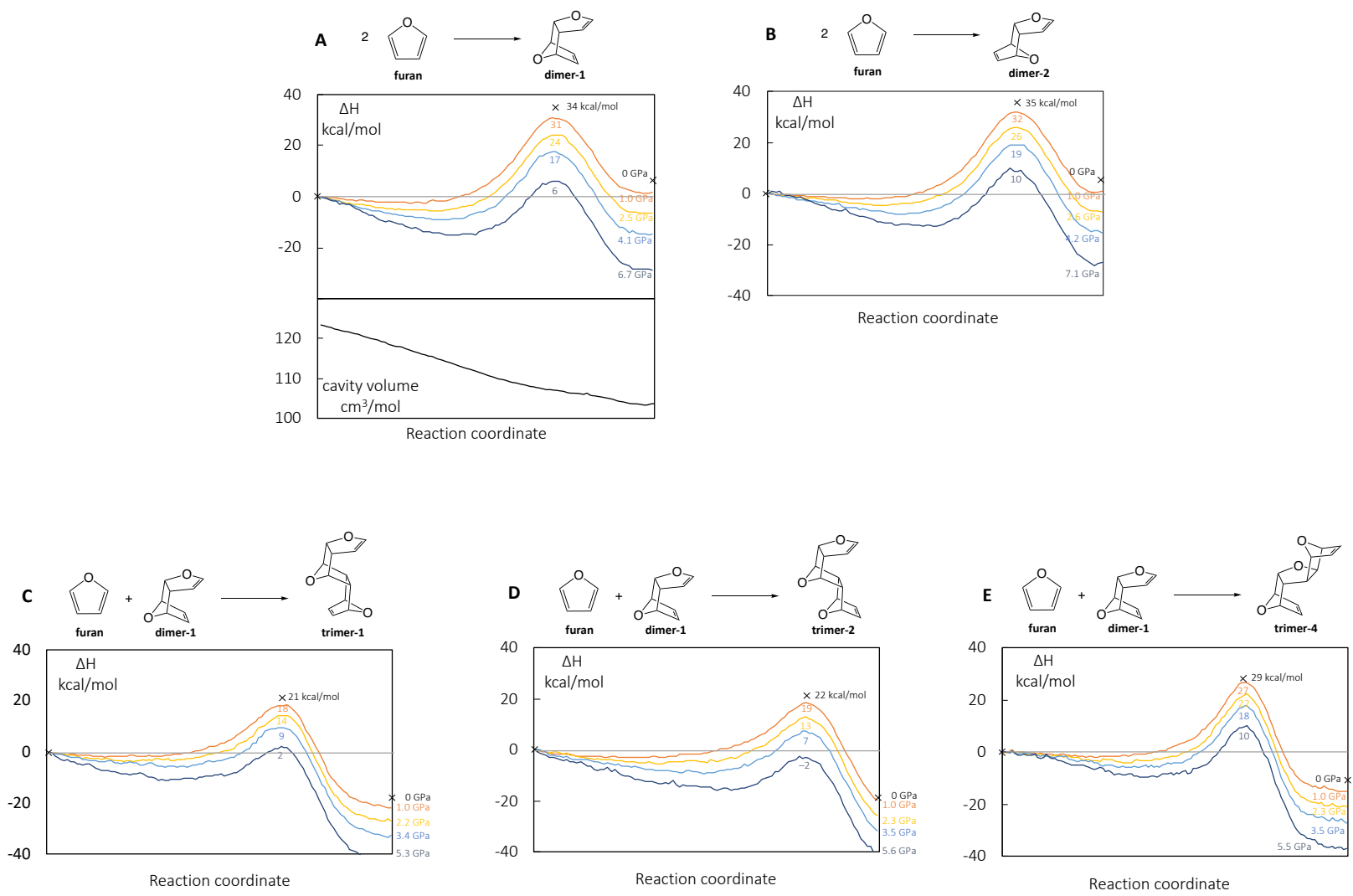

Figure 4. Enthalpic reaction profiles of two initiation reactions $\mathbf{A}$ and $\mathbf{B}$ (top) and three dimer-to-trimer reactions $\mathbf{C}-\mathbf{E}$ (bottom) for furan $4+2$ polymerization at different pressures, calculated by the XP-PCM method. The volume profile for the reaction $\mathbf{A}$ is shown.

One interesting phenomenon from the calculations is the appearance of a local minimum in the enthalpic profiles about halfway between the reactant and the transition state; this entrance channel minimum shifts towards the transition state as 
the pressure increases. A reactant complex before bond formation is intriguing. Is it simply the result of proximity, a $\mathrm{p} \Delta \mathrm{V}$ effect of complexation? Or is it intrinsic to the electronic progress of the reaction- the outcome of dispersion forces and enhanced electron transfer between diene and dienophile in the early stages of the reaction? This will be studied further.

In order to calculate the activation volume of a reaction (Table 1), the enthalpic barriers are plotted against the pressures and the $\Delta \mathrm{H}^{\ddagger}$-p relationship is fitted to a line (linear relationship). The slope of the fitted line gives the activation volume, according to $\Delta \mathrm{V}^{\ddagger}=d \Delta \mathrm{H}^{\ddagger} / d \mathrm{p} .{ }^{12}$ Note that for the calculations of activation volumes, the enthalpic barriers at different pressure are computed from the enthalpic differences between the transition states and the enthalpies of isolated reactant molecules, not the reactant complexes (see Figure-S3 in SI for the pressure-barrier data used in activation volume calculations). This is to ensure a common reference (isolated reactants) in the comparison of the endo/exo pathways.

Table 1. Computed activation volumes using the XP-PCM method at the $\omega$ B97xd/def2TZVP level.

\begin{tabular}{|l|l|l|l|}
\hline Reaction & Description & $\begin{array}{l}\Delta \mathrm{V}^{\ddagger} \text { in } \mathrm{cm}^{3} / \mathrm{mol} \text { for } \\
\text { furan }\end{array}$ & $\begin{array}{l}\Delta \mathrm{V}^{\ddagger} \text { in } \mathrm{cm}^{3} / \mathrm{mol} \text { for } \\
\text { thiophene }\end{array}$ \\
\hline A & Initiation via ts1-1 (endo) & -27.5 & -25.6 \\
\hline B & Initiation via ts1-2 (exo) & -26.1 & -25.5 \\
\hline C & $\begin{array}{l}\text { dimer-1 to trimer-1 via } \\
\text { ts2-1 (endo) }\end{array}$ & -27.4 & -26.8 \\
\hline D & $\begin{array}{l}\text { dimer-1 to trimer-2 via } \\
\text { ts2-2 (exo) }\end{array}$ & -26.3 & -26.1 \\
\hline E & $\begin{array}{l}\text { dimer-1 to trimer-4 via } \\
\text { ts2-4 }\end{array}$ & -26.1 & -25.6 \\
\hline
\end{tabular}


As shown in Table 1, for the furan system, the endo cycloadditions are calculated to have more negative activation volumes than the exo cycloadditions, -27.5 vs -26.1 $\mathrm{cm}^{3} / \mathrm{mol}$ in the initiation reaction, and $-27.4 \mathrm{vs}-26.3 \mathrm{~cm}^{3} / \mathrm{mol}$ in the dimer-to-trimer reactions. However, for the thiophene system, the endo and exo cycloadditions have almost the same activation volume of about $-26 \mathrm{~cm}^{3} / \mathrm{mol}$ in the initiation reaction, and the difference is only $0.7 \mathrm{~cm}^{3} / \mathrm{mol}$ in the dimer-to-trimer cycloaddition. The activation volume difference in endo/exo cycloadditions from furan to thiophene is not large, but it is significant $\left(1 \mathrm{~cm}^{3} / \mathrm{mol}\right.$ difference in activation volume leads to $2.4 \mathrm{kcal} / \mathrm{mol}$ difference in activation enthalpy at $10 \mathrm{GPa}$ ).

We try to understand the difference in endo/exo cycloadditions by thinking of the relative sizes of the atoms/groups on the left and right-hand sides of the bridging bonds in the bottom rings in the transitions state structures in Scheme 2.

Scheme 2. The endo and exo configurations of the [4+2] cycloaddition TS.

furan
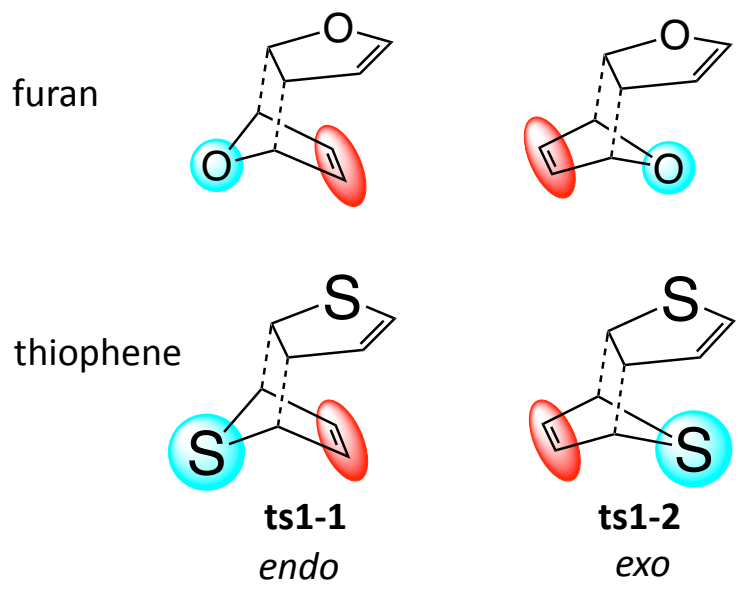

In the furan cycloadditions, since the $\mathrm{O}$ atom is small, the double bond in the bottom ring should be bulkier (this a qualitative judgment, based on chemical intuition) than the $\mathrm{O}$ atom; therefore, the endo TS, in which the bulkier double bond in 
the bottom ring interacts with the top ring, appears to be more compact than the exo TS. This is probably the reason that the endo cycloaddition of furan has a more negative activation volume than the exo cycloaddition. However, for thiophene, the bigger $\mathrm{S}$ atom results in a smaller steric difference between the $\mathrm{S}$ atom and the double bond in the bottom ring, so that the steric congestion with the top ring is similar in both the endo and exo cycloaddition transition states. Consequently, the two TSs have similar activation volumes.

The above discussion answers the first question we posed in the beginning of this section. Now we address the second question: whether pressure would have a different effect on furan cycloadditions contrasted with thiophene cycloadditions. Table 1 shows that for all the five cycloadditions, the computed activation volumes for the furan reactions are more negative than those for the thiophene reactions. This suggests that pressure would favor the cycloaddition polymerization of furan over thiophene. Considering that the intrinsic 1 atm reaction barriers of the furan cycloadditions are lower than those of the corresponding thiophene reactions, the furan polymerization is expected to begin at a lower pressure and proceed faster than its thiophene analog. Experimentally, nanothread formation from furan starts at $10 \mathrm{GPa},{ }^{11}$ only half of the onset pressure of $20 \mathrm{GPa}^{8}$ for thiophene nanothread formation, as indicated by Raman spectroscopy.

Finally, for the chain elongations in "a different direction", via ts2-4 (reaction E), the computed activation volumes are slightly less negative than for the other chain lengthening reactions $\mathbf{C}$ and $\mathbf{D}$; pressure is unlikely to favor this type of bidirectional chain-growth. 


\section{Lone pair repulsion}

One important feature of the syn structure of furan/thiophene nanothreads, compared with the other two structures in Figure 1, is that the syn structure has a chain of nonbonded chalcogens in close proximity. Are there some relevant models we might think of in this context? Hypothetical polyketone $(\mathrm{CO})_{\mathrm{n}}$ is one example (Scheme 3), calculated to prefer a helical structure. ${ }^{21}$ Polythiene $(C S)_{n}$, a hypothetical

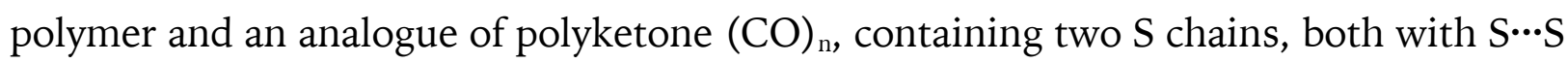
Interactions, has been suggested. ${ }^{22}$ Extended $(\mathrm{CO})_{\mathrm{n}}$ and $(\mathrm{CS})_{\mathrm{n}}$ polymers show a geometry that we will see is analogous to that of all-syn thiophene and furan nanothreads, so it is useful to look at these structures in context. ${ }^{23}$

Scheme 3. Polyketone and polythiene.

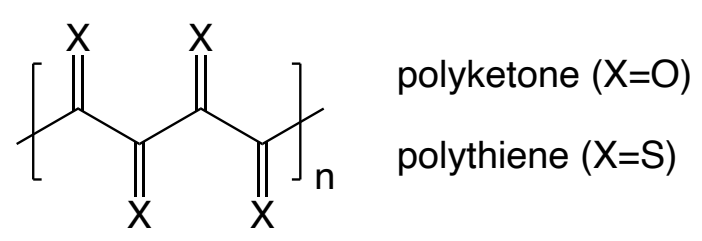

As shown in Figure 1, the neighboring nonbonded $\mathrm{O} \cdots \mathrm{O}$ ( or $\mathrm{S} \cdots \mathrm{S}$ ) distance in the relaxed syn thread (subject to periodic boundary condition, one monomer unit per cell) is about $2.6 \AA$ (or $2.8 \AA$ ), shorter than the typical van der Waals distance between two nonbonded O (or S) of 3.0-3.4 $\AA$ (or 3.6-4.2 $\AA$ )..$^{24,25}$ This means that repulsive forces between adjacent chalcogens are likely. From an orbital point of view (Figure 7 inset), the $\mathrm{X} \cdots \mathrm{X}(\mathrm{X}=\mathrm{O}$ or $\mathrm{S})$ repulsion mainly arises from the unfavorable 4-electron interaction between high-lying neighboring $\mathrm{O} 2 \mathrm{p}_{z}$ (or $\mathrm{S} 3 \mathrm{p}_{z}$ ) lone pair orbitals, where $z$ is the axial direction of the thread. These fragment AOs are likely the highest occupied 
MOs of the polymers. These destabilizing interactions are sometimes termed Pauli repulsions in the literature.

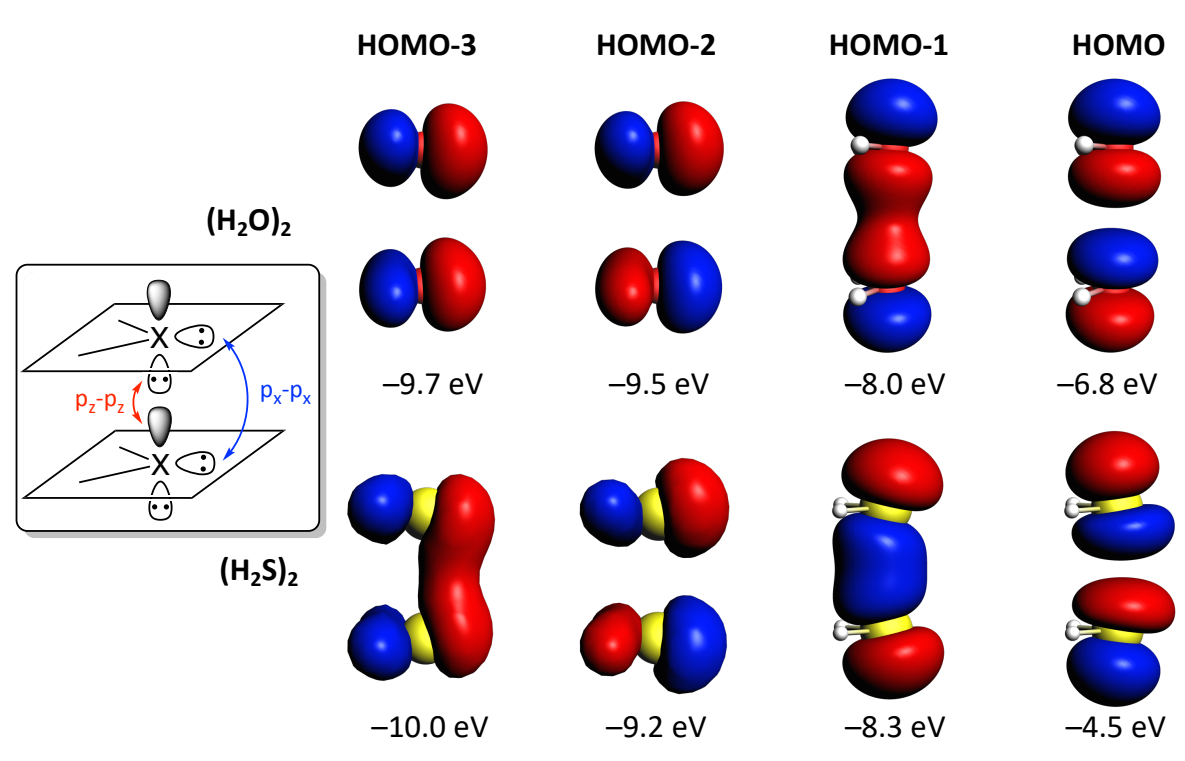

Figure 5. Lone pair orbitals with computed energies of two molecules $\left(\mathrm{H}_{2} \mathrm{O}\right)_{2}$ or $\left(\mathrm{H}_{2} \mathrm{~S}\right)_{2}$, stacked on top of each other the same way as in the syn thread, at 2.6 and $2.8 \AA$ separation, respectively.

Constrained geometry dimers of $\mathrm{H}_{2} \mathrm{O}$ and $\mathrm{H}_{2} \mathrm{~S}$ may serve as models for the lone pair interaction. Figure 5 shows the lone pair orbitals of $\left(\mathrm{H}_{2} \mathrm{O}\right)_{2}$ and $\left(\mathrm{H}_{2} \mathrm{~S}\right)_{2}$ pushed on each other in a geometry that simulates the $\mathrm{X} \cdots \mathrm{X}$ approach in the all-syn isomer. The highest occupied molecular orbital (HOMO) and HOMO-1 are the $2 / 3 \mathrm{p}_{z}$ combinations; the HOMO-2 and HOMO-3 are the $2 / 3 \mathrm{p}_{\mathrm{x}}$ combinations with some $2 / 3 \mathrm{~s}$ admixture. The repulsion of the filled orbitals is clear -- the $1.2 \mathrm{eV}$ splitting of HOMO and HOMO- 1 , the antisymmetric and symmetric $3 p_{z}$ lone pair combinations, is $3.8 \mathrm{eV}$ for thiophene, $1.2 \mathrm{eV}$ for furan. The $\mathrm{p}_{\mathrm{x}}$-type lone pairs interact much less, as expected. 
Returning to the polymers, in the anti isomer, the anti relationship of adjacent chalcogens removes the above lone-pair repulsion (though perhaps some inter-ring repulsion between the lone pair and $\mathrm{C}-\mathrm{C}$ bonds remains). In the syn-anti isomer, most of the lone-pair repulsion is removed; the remaining $\mathrm{X} \cdots \mathrm{X}$ pairs suffer smaller repulsion, especially after eventual "curving" of the polymer is allowed in a geometry optimization, creating an "S-curve" shape to the polymer overall, increasing the $\mathrm{X} \cdots \mathrm{X}$ separation.

The difference in the strength of lone-pair repulsion in the three threads is reflected by the computed relative energies (idealized geometries still maintained). As shown in Figure 6, the syn thread is computed to be, as expected, the highest in energy among the three isomers. The tiny energy differences between the anti and syn-anti structures indicate that the lone-pair repulsion is almost completed removed in the synanti thread, as it is in the anti thread. 

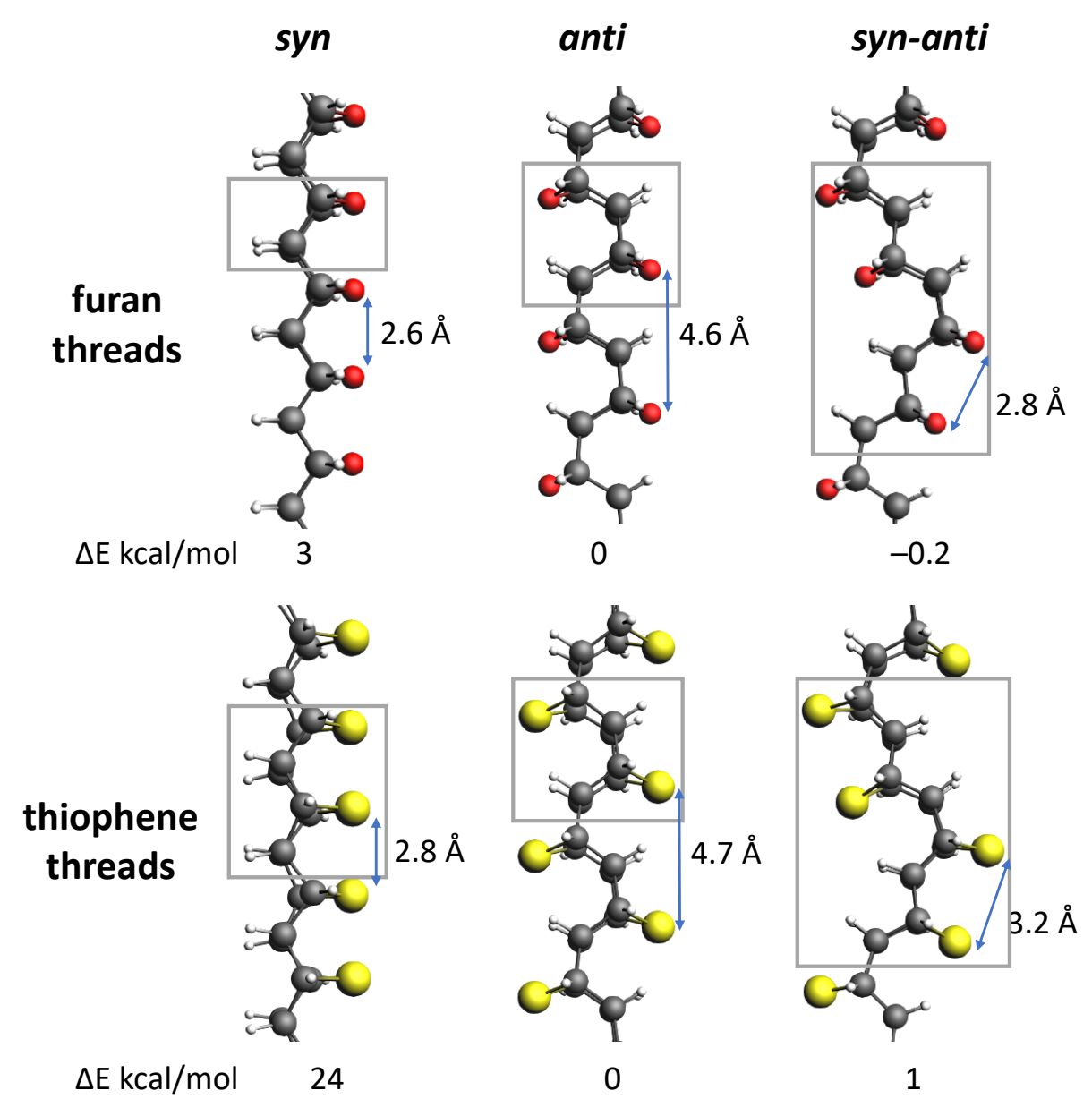

Figure 6. Optimized structures (under periodic boundary conditions) and relative energies of three types of furan and thiophene threads, in idealized conformations. Unit cells are indicated by grey boxes. Relative energies are in kcal per mole of $\mathrm{C}_{4} \mathrm{H}_{4} \mathrm{X}$ $(\mathrm{X}=\mathrm{O}$ or $\mathrm{S})$.

Two things we would also like to understand are (a) the higher energy of the syn relative to the anti thread for thiophene $(24 \mathrm{kcal} / \mathrm{mol})$ than for furan $(3 \mathrm{kcal} / \mathrm{mol})$, and (b) the larger energy difference between the syn and anti structures for the threads in Figure $6-3$ and $24 \mathrm{kcal} / \mathrm{mol}$ for furan and thiophene, respectively-compared with 
those for the oligomers in Figure 3, which are within $2 \mathrm{kcal} / \mathrm{mol}$. The computed relative stability of various isomers are consistent with previous calculations. ${ }^{26}$

The former is due to the larger atomic size of $\mathrm{S}$ compared to $\mathrm{O}$, and consequently a greater overlap and larger repulsion between the $\mathrm{S}$ atoms than between the $\mathrm{O}$ atoms, in the syn threads at similar distances. The larger energy difference between the syn and anti structures arises from the periodic boundary conditions (PBC) enforced in the calculations in Figure 6. In the constraint-free syn oligomers in Figure 3 , the threads are able to and do curve, in order to increase the distance between neighboring chalcogen atoms. However, in the calculation of infinite-length threads with a minimal unit cell, $\mathrm{PBC}$ force the syn structure to be "straight", resulting in a large repulsion between the chalcogens, thus raises the relative energy of the syn thread.

The straightness constraint of PBC may seem like an unrealistic choice. But we should take it seriously, for the constraints operative in the compressed solid state may in fact be a more realistic picture of what such a polymer faces in the solid. As we will show in much more detail in the next section, in gas phase, constraint-free syn threads curve, in order to relieve the repulsion between chalcogens. In the solid state, the extent of arching depends on the competition between efficient packing (being straight) and electronic energy relaxation (being bent), which we attempt to quantify in the next section.

\section{Thread arching}

To probe the proclivity to arching or bending of the syn thread, we cut out segments of different lengths from a straight syn thread that was relaxed under periodic 
boundary conditions with optimized axial length, terminate the finite-length oligomers with hydrogens, and relax the geometries without periodic boundary condition. Shown in Figure 7 are two segments of furan and thiophene threads comprising 10 molecular units with 4 additional hydrogens at the termini. As expected, the threads arc.

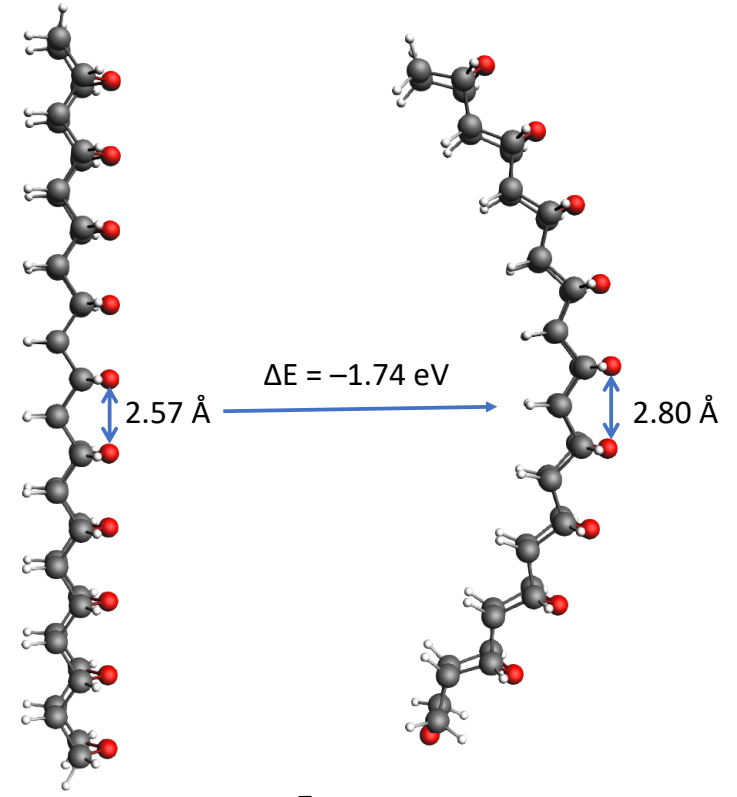

Furan

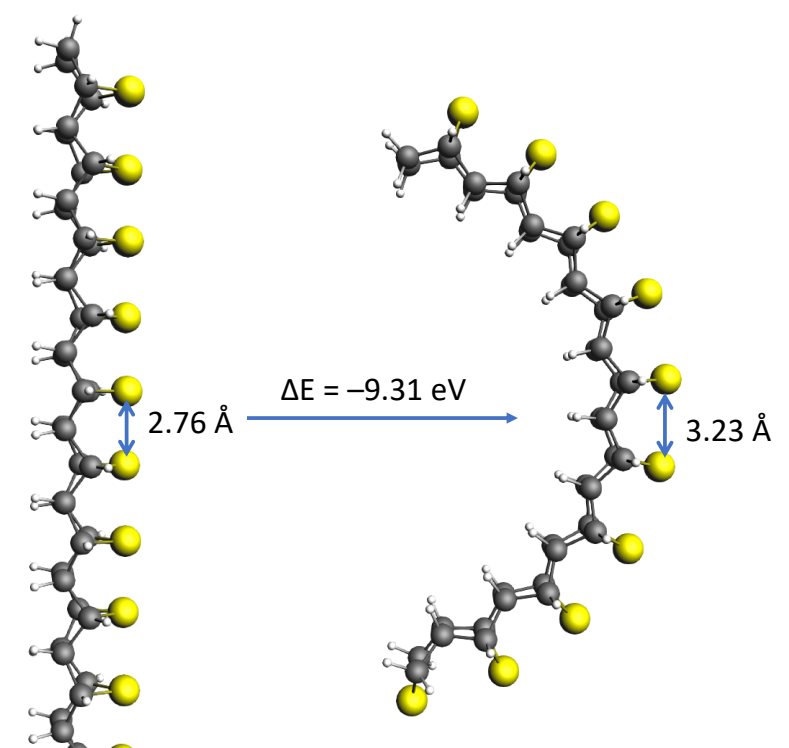

Thiophene

Figure 7. Structural and energy changes upon relaxation of two 10-unit segments of the straight syn threads of furan and thiophene, calculated at the PBE-D3BJ/6-31G(d) level of theory.

The relaxed thiophene syn thread segment shows a larger S ‥S spacing of $3.2 \AA$ and a larger relaxation energy of $9.31 \mathrm{eV}$, compared with the $2.8 \AA \mathrm{A} \mathrm{O} \cdots \mathrm{O}$ separation and the $1.74 \mathrm{eV}$ relaxation energy for the syn furan thread segment. The larger curvature in the thiophene thread is consistent with the larger S lone pair repulsion we adduced above. 
The relaxed structures of segments of different lengths are given in Figure S4 and Figure S5 in SI. Similar curvature was observed for furan (or thiophene) threads with different lengths. The relaxation energy decreases as the thread lengthens and converges to about $0.13 \mathrm{eV} / \mathrm{C}_{4} \mathrm{H}_{4} \mathrm{O}$ for the furan threads and $0.91 \mathrm{eV} / \mathrm{C}_{4} \mathrm{H}_{4} \mathrm{~S}$ for the thiophene threads (Figure S4 and S5 in SI), slightly smaller than corresponding values for the 10-unit segments in Figure 7.

Subtracting the $0.13 \mathrm{eV} / \mathrm{C}_{4} \mathrm{H}_{4} \mathrm{O}$ and $0.91 \mathrm{eV} / \mathrm{C}_{4} \mathrm{H}_{4} \mathrm{~S}$ relaxation energies from the energy difference between the syn and the anti threads $\left(0.14 \mathrm{eV} / \mathrm{C}_{4} \mathrm{H}_{4} \mathrm{O}\right.$ and 1.02 $\mathrm{eV} / \mathrm{C}_{4} \mathrm{H}_{4} \mathrm{~S}$ ) in Figure 6, one gets 0.01 and $0.11 \mathrm{eV}$, numbers close to 0 . The implication is that fully relaxed, bent syn structures compare in stability to anti and syn-anti structures, all having little-to-no $\mathrm{X} \cdots \mathrm{X}(\mathrm{X}=\mathrm{O}$ or $\mathrm{S})$ repulsion. Note that lacking $\mathrm{X} \cdots \mathrm{X}$ repulsion, the anti threads do not arch and are inherently straight; for the syn-anti thread, the adjacent X $\cdots X$ pairs do curve the thread locally, but their alternating sense of curving on both sides along the thread leads to a globally straight structure.

Arching in this manner, driven by a single atomic stack of "lone-pair repellers," requires a structure that is rigid (to define a minimum around which to arch), yet relatively thin (to enable noticeable arching) —again, a unique feature that distinguishes nanothreads from traditional polymers and nanotubes. The strain introduced into the carbon backbone by arching (relative to a hypothetical structure with no lone-pair repulsion) is modest, as reflected by the similar stability of the arched syn structure and the straight anti structure. This reflects the presence of two, not three new bonds introduced by polymerization along the thread axis: whereas a triply linked column does not easily accommodate arching, a doubly-linked ladder (such as we have in the furan- or thiophene-derived threads) has one easy direction of 
arching that does not require compression or extension of the covalent bonds associated with the polymerization.

The synthesized furan nanothread sample showed a clear six-fold diffraction pattern at $1.5 \mathrm{GPa}$ during pressure release from $15 \mathrm{GPa} .{ }^{11}$ These diffraction spots are among the sharpest observed for nanothreads synthesized from various precursors. More intriguingly, when the pressure is released to ambient, these sharp diffraction spots of furan nanothreads becomes fuzzy, diffusing in both the d-spacing and the azimuthal angle. This degradation of diffraction quality from a few GPa to $1 \mathrm{~atm}$ is the most severe among of nanothread samples reported so far. ${ }^{11}$ A plausible explanation is that some of the synthesized threads have short segments of syn configuration. We speculate that these syn segments (or perhaps other types of defective segments not yet identified) are forced to be straight under high pressure to bring about a more favorable packing, but then curve upon the release of the last $1.5 \mathrm{GPa}$, disrupting the crystalline packing and thus broadening the diffraction spots.

Can one quantify the competition between the efficient packing of straight threads and the favorable reduction of lone-pair repulsion in bent or arched threads? We computed the van der Waals cohesion energy at $1 \mathrm{~atm}$ for individual furan syn threads packing in solid to be $0.22-0.34 \mathrm{eV} / \mathrm{C}_{4} \mathrm{H}_{4} \mathrm{O}$, depending on the packing geometry. ${ }^{11}$ The packing geometries were optimized at 1 atm as described in ref 11 . For individual threads, a large tetragonal unit cell $(20 \AA \times 20 \AA \times c \AA$, where $\mathrm{c}$ is the axial periodicity of the thread) was used in the geometry optimization. The cohesion energy is computed as the energy difference between the packed threads and isolated individual threads. 
As discussed above, the arching relaxation energy for furan syn thread is 0.13 $\mathrm{eV} / \mathrm{C}_{4} \mathrm{H}_{4} \mathrm{O}$, smaller than the vdW cohesion energy. In the solid state, complete arching relaxation, as can be simulated in gas-phase calculations, is unlikely, as is the total loss of the cohesive energy; instead, partial relaxation will be attained at the expense of a fraction of the cohesion energy. The arching and cohesion energies being of the same order suggest that partial arching of syn segments could occur during the pressure release from $1.5 \mathrm{GPa}$ to $1 \mathrm{~atm}$. We note here that our previous packing simulations suggested that the anti furan threads are a more likely candidate than the syn threads, based on the match to experimentally-measured d-spacings; anti threads are inherently straight and do not arch. Segments of syn thread could be a minor component in the synthesized sample. Other potential structural sources of localized arching, such as point defects within anti threads, may experience a similar counterbalance of cohesive and arching energies in the solid state. Indeed, solid state NMR studies, reported elsewhere, ${ }^{16}$ give a clear indication of segments of these structures with $\mathrm{C}-\mathrm{C}$ unsaturation, i.e., alkene units.

A bit of prospecting before ending this section. Individual syn furan threads, if dispersed in solution, are likely to acquire their native curvature (as modulated by solvent interactions). Depending on the length of the threads, they may bend into an arc or curl up into a coil (Figure 8). When the length of the thread is appropriate, one can think of functionalizing and cross-coupling the termini to make a macrocycle (Figure 8). A similar cyclic structure derived from benzene $4+2$ polymer was previously proposed by Grimme. ${ }^{27}$ These hypothetical cyclic and coil structures of furan/thiophene polymers have a hydrophobic interior and hydrophilic exterior, a feature that may find applications in separation, catalysis, or perhaps drug delivery. In addition, these structures should have small overall dipole moments because of the cylindrically symmetric arrangement of the polar $\mathrm{C}-\mathrm{O} / \mathrm{C}-\mathrm{S}$ bonds. Also of interest 
would be the response of the orientations of the $\mathrm{C}-\mathrm{O} / \mathrm{C}-\mathrm{S}$ bonds in an electric field, and the corresponding change of the coil structure-whether lengthening or shortening, expanding or contracting. These remain interesting topics for future studies.
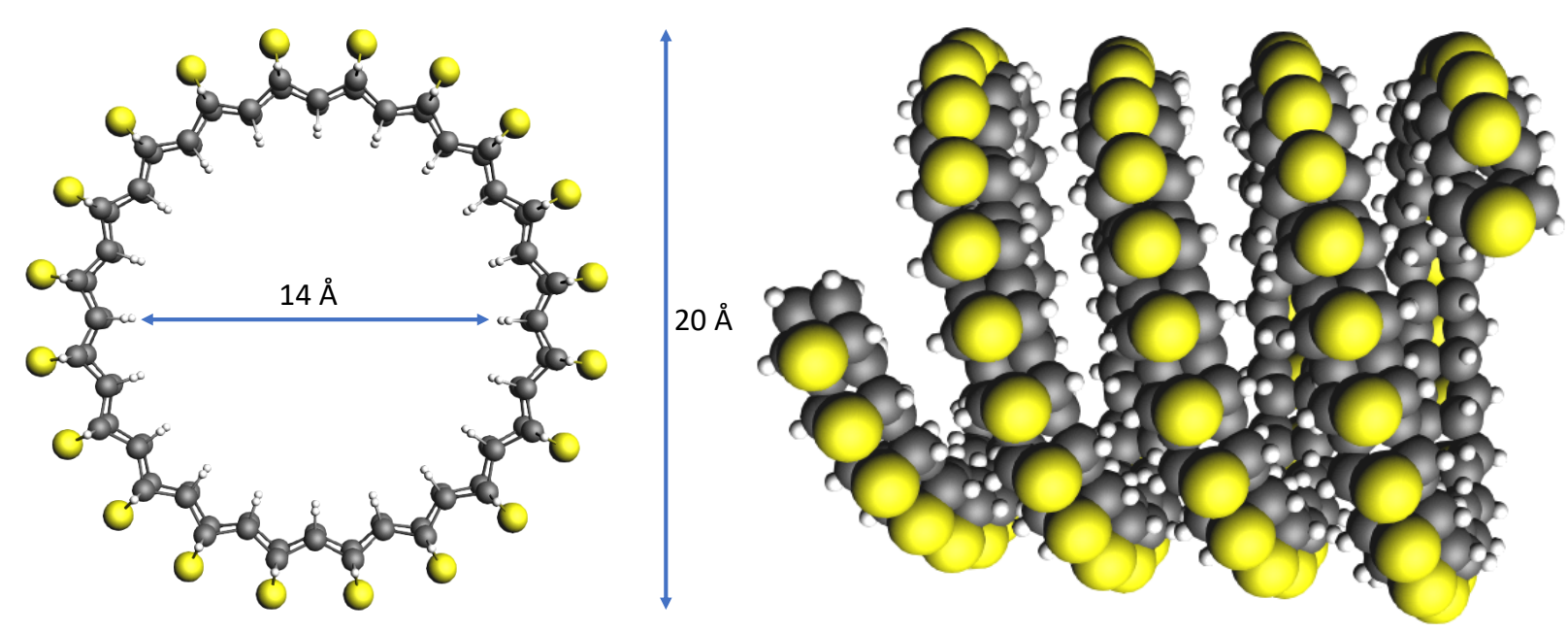

Figure 8. A hypothetical ring and a coil structures built from curved syn thiophene nanothreads.

\section{Thread twisting}

For a syn thread, there are two relaxation modes that increase the distance between chalcogen atoms and reduce the repulsion between them (Figure 9). "Axial " relaxation is the more effective way to increase the $\mathrm{X} \cdots \mathrm{X}(\mathrm{X}=\mathrm{O}$ or $\mathrm{S})$ distances under free boundary conditions; it causes thread arching, as discussed above. Lateral relaxation, where the chalcogen atoms move to avoid each other sideways, leads to 
thread twisting and may be favored in the dense solid state where interthread cohesion suppresses large-scale arching. Different kinds of twisting are possible, depending on the unit cell size used in the calculation. Zig-zag twisting with a two or four-ring repeating unit is shown in Figure 9. In the extreme case, where all chalcogen atoms "twist" in the same direction, a helical chain of chalcogens forms.
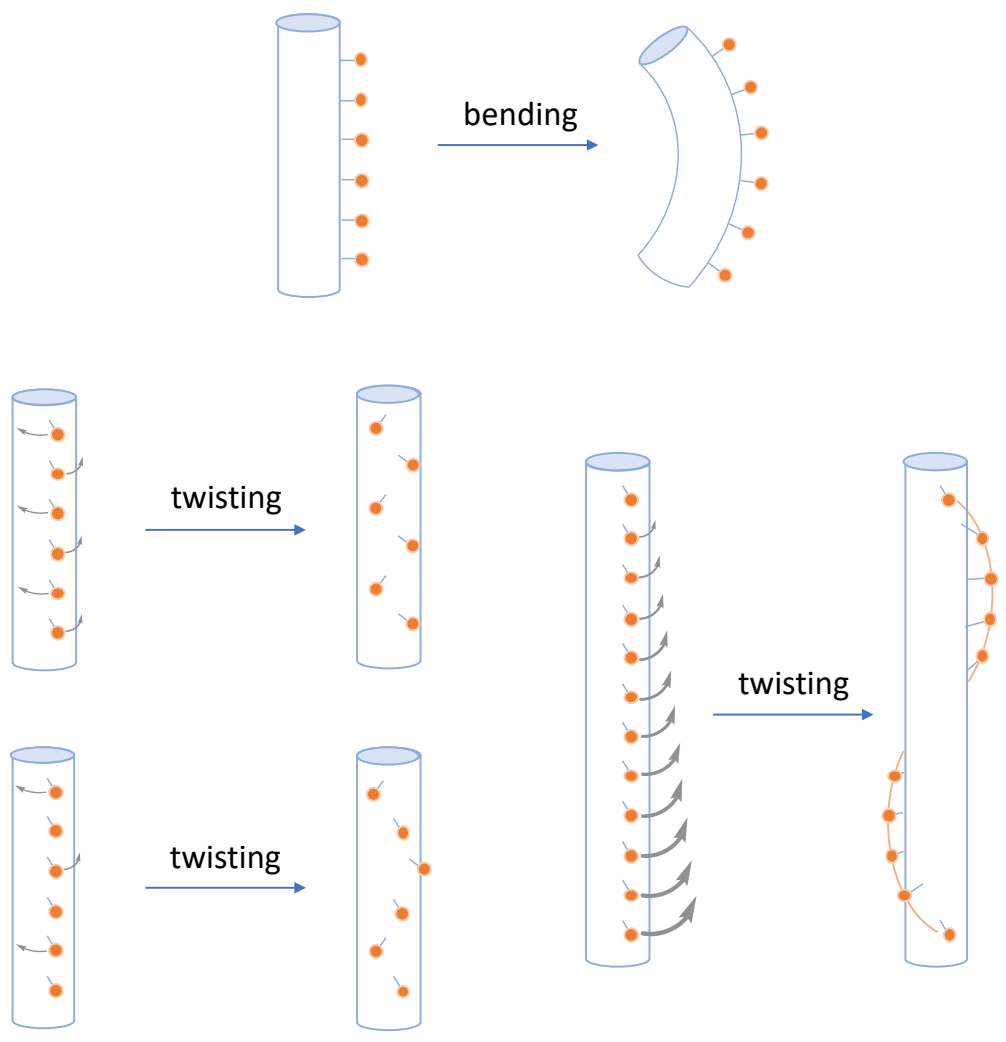

Figure 9. Illustration of thread bending (arching) and twisting as results of axial and lateral relaxations of the lone pair repulsion between the chalcogens (orange balls) in the syn thread.

To probe the twisting behavior of the syn thread (Figure 10), we started with the straight structure of a thiophene thread (one $\mathrm{C}_{4} \mathrm{H}_{4} \mathrm{~S}$ per unit cell; $\mathrm{Z}=1$ ), gradually increased the unit cell size, and moved the $S$ atoms sideways to initiate the types of 
twisting shown in Figure 9, and then relaxed the structures. In the calculations, we kept the unit length (the length per $\mathrm{C}_{4} \mathrm{H}_{4} \mathrm{~S}$ unit) the same for all threads, in order to maintain similar axial strain in the backbone. If the length constraint is lifted, some threads will shrink in length and expand in width into a coil structure.

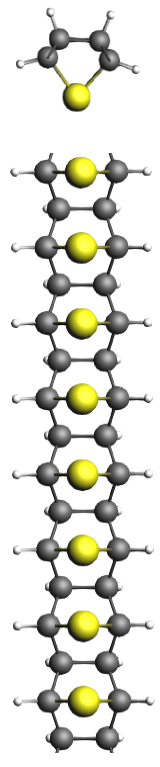

$\mathrm{Z}=1$

S...S distance (Å) $2.63 \AA$

$\triangle \mathrm{E}\left(\mathrm{eV} / \mathrm{C}_{4} \mathrm{H}_{4} \mathrm{~S}\right) \quad 0$
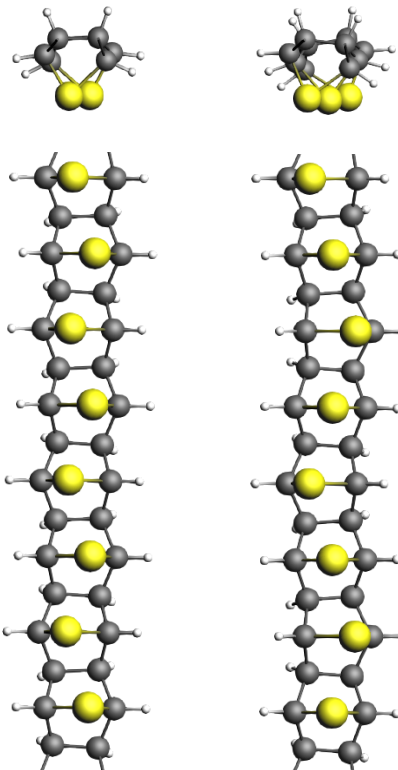

$\mathrm{Z}=2$

$2.75 \AA$

$-0.16$

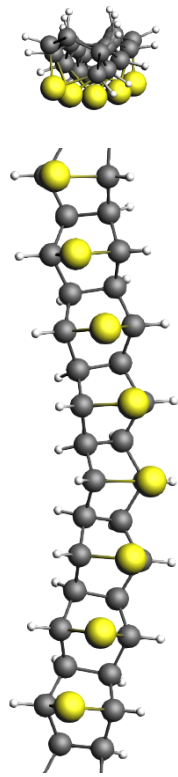

$Z=8$

$2.78 \AA$

$-0.22$
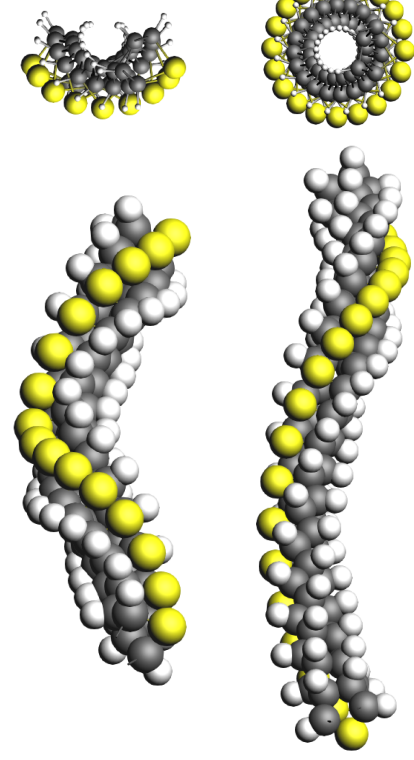

$\mathrm{Z}=20$

$2.86 \AA$

$-0.32$

Figure 10. Top and side views of the twisting deformation of the syn thiophene nanothread. All threads have the same unit length. The last two structures are viewed slightly tilted, so that they appear shorter than the others in the sideview. Unit cell sizes, S...S distances, and relative energies are given.

The straight thiophene syn thread $(Z=1)$ has the strongest lone pair repulsion and the highest relative energy. All of the relaxations we study are stabilizing. In the 
$Z=2$ to $Z=16$ structures, there is a zig-zag twisting pattern, whereas the $Z=20$ structure is produced by twisting the repeating units in one direction only, forming a helix. As the Z-number of the twist increases, the S...S distances increases and the relative energy decreases, appearing to converge to $2.86 \AA$ and $-0.32 \mathrm{eV} / \mathrm{C}_{4} \mathrm{H}_{4} \mathrm{~S}$, respectively, at $Z=20$. Twisting also increases the width of the thread, especially for those with large unit cells. Expanding in width actually allows some arching to be developed in the structure. This is why the S...S distance keeps increasing with unit cell size, despite of the fact that the twist angle appears to be similar in each thread. The larger the unit cell, the more freedom the structure has to relax the lone pair repulsion, and the lower the relative energy of the relaxed structure.

It would not be difficult to imagine that stretching the helical thread $(Z=20)$ will straighten the thread, shorten the S $\cdots S$ distances, and raise the energy of the thread. Or conversely, shortening the length of the same thread will induce more arching and expansion in width, which will finally lead to the coil structure shown in Figure 8 (see Figure S7 in SI for some intermediate structures during coiling). The energy change from the $Z=20$ helical structure to the coil structure is as large as 0.9 $\mathrm{eV} / \mathrm{C}_{4} \mathrm{H}_{4} \mathrm{~S}$, indicative of the large instability of the former structure. However, the constraint of the solid-state packing and high pressure are unlikely to allow for such a large extent of twisting and expansion in width (as in the $\mathrm{Z}=20$ thread). But small twisting (as in the $\mathrm{Z}=2$ thread) may still be possible and larger-scale twisting and coiling, as noted earlier, would be possible in solution, likely with interesting electromechanical couplings.

Our calculations showed that the syn furan thread does not twist. The oxygen atoms stay "in the middle" of the thread during the relaxation, irrespective of the unit cell sizes. This suggests that the lone-pair repulsion between the $\mathrm{O}$ atoms in the syn 
furan thread is small, which is consistent with the small energy difference between the syn and the anti furan threads (3 kcal/mol in Figure 6). Different interplays of twisting, coiling, and arching should be anticipated for other thread types, produced by judicious choices of precursor that yield thread backbones with the requisite symmetry-breaking and orbital interactions to activate these various modes.

\section{Conclusions}

This work is motivated by the recent synthesis of furan and thiophene nanothreads under slow compression. We set out to understand the $20 \mathrm{GPa}$ difference in the synthetic pressure (both the onset and maximum pressures) in the two nanothread syntheses. By calculating the 1 atm reaction profiles of furan and thiophene $[4+2]$ cycloadditions for the first two polymerization steps, we found that

(1) the furan reacts with a lower barrier than thiophene; this is explained by the reduced aromaticity of furan compared with thiophene,

(2) the endo and exo pathways of the [4+2] cycloadditions have very similar barriers at 1 atm, with endo chain extension being slightly favored by $<1$ $\mathrm{kcal} / \mathrm{mol}$,

(3) while the dimers are a few $\mathrm{kcal} / \mathrm{mol}$ less stable than the reactant molecules, the trimers are already more stable than the reactants.

These results along with the high 1 atm barriers suggests that polymers made at high pressure will be stable against decomposition to monomers when recovered to $1 \mathrm{~atm}$. 
We next examined the effect of pressure on these cycloadditions, using the recently-developed XP-PCM method. The enthalpic profiles at high pressures and activation volumes of five representative reactions of the [4+2] polymerization were computed, which show that

(1) pressure decreases the enthalpic barriers of all these cycloadditions due to the volume reduction nature of these bond-formation cycloadditions;

(2) the endo cycloadditions are favored over the exo cycloadditions by pressure for furan but much less so for thiophene. Inspection of the endo and exo configurations of the cycloaddition transitions state suggests that the larger size of the $S$ atom, compared with the $\mathrm{O}$ atom, results in a relatively small steric difference in the moieties in the diene that define the endo/exo configuration (the chalcogen atom and the double bond on opposite sides of the bridging $\mathrm{C}-\mathrm{C}$ bonds, see Scheme 2). Such small steric differences in furan cycloaddition TS lead to similar volumes of the endo and exo TS, so that pressure has a smaller effect on the endo/exo selectivity in thiophene cycloadditions;

(3) the furan cycloadditions have more negative activation volumes than the thiophene cycloadditions, which, along with the computed lower 1 atm barriers for furan cycloadditions, rationalizes the lower synthetic pressure for furan nanothreads;

(4) bidirectional growth of the polymer is not favored under pressure due to its less negative activation volume. The last point suggests that furan or thiophene nanothread ends that are examined by scanned probes may be able to interrogate the geometry of thread nucleation. 
We then go on to understand the structures and relative stabilities of the syn, syn-anti, and anti furan/thiophene nanothreads formed from a [4+2] cycloaddition pathways. The higher energies of the syn threads are rationalized based on the lone pair repulsions between the closely positioned chalcogens forced by the structure. The thiophene syn thread experiences larger lone-pair repulsion because of the bigger size of S compared to O. A straight syn thread curves or arches due to such lone pair repulsion. Depending on the length and environmental constraints, a straight syn thread could undergo either axial (curving) or lateral (twisting) relaxations, or both. The structures and their energies during these relaxations were traced, which led to the suggestion of two hypothetical structures (a ring and a coil) for thiophene syn thread. Lacking the lone pair repulsions, the anti thread does not curve and is inherently straight; with most of the lone pair repulsion removed, the syn-anti thread assumes locally bent but globally straight structure. The relaxation energy and the adhesion energy of individual syn threads were calculated to be on the same order, suggesting possible competition between the two modes in the synthesized nanothread sample.

\section{Computational Methods}

The potential energy surface calculations in Figure 3 were done using the Gaussian $09^{28}$ package of programs. Geometries were optimized at the B3LYP ${ }^{29} / 6$ $31 \mathrm{G}(\mathrm{d})^{30,31,32}$ level of theory. Singlet-point calculations at the DLPNO-CCSD(T) ${ }^{33} / \mathrm{cc}-$ pVTZ $^{34,35}$ (also with the cc-pVTZ/C ${ }^{36,37}$ auxiliary basis) levels were performed on the B3LYP-optimized geometries, using the ORCA $4^{38,39}$ package of programs.

The enthalpic profiles of the cycloadditions under pressure in Figure 4 were computed by the eXtreme Pressure Polarizable Continuum Model (XP-PCM) ${ }^{40}$ method, 
at the $\omega \mathrm{B} 9 \mathrm{xd}^{41} / \mathrm{def} 2-\mathrm{TZVP}{ }^{42,43} /$ Fit level with density fitting, using intrinsic reaction coordinate (IRC) paths calculated at the B3LYP/6-31G(d) level. The XP-PCM computations were done with a Julia script in junction with Gaussian 09. In order to choose a proper density functional for the XP-PCM calculations, the performance of five functionals were benchmarked against the DLPNO-CCSD(T)/cc-pVTZ and $\operatorname{CCSD}(\mathrm{T})^{44,45} / \mathrm{cc}-\mathrm{pVTZ}$ single-point results (Figure S1 in SI); $\omega \mathrm{B} 97 \mathrm{xd} / \mathrm{def} 2-\mathrm{TZVP} /$ Fit seems to be the best among those tested, and is chosen for the XP-PCM calculations.

The orbitals in Figure 5 were computed with the $\mathrm{ADF}^{46}$ package, using the SCCDFTB $^{47}$ method with the QUASINANO2015 $5^{48,49}$ parameter set. The one-dimensional structures in Figure 6 and Figure 10 were calculated using the VASP ${ }^{50}$ package with the $\mathrm{PBE}^{51,52}$ functional, D3-BJ ${ }^{53}$ dispersion correction, and a plane wave basis set with 600 eV cutoff energy. The thread is put in a tetragonal unit cell with dimensions $20 \AA \times 20$ $\AA$ × c $\AA$, where $\mathrm{c}$ is the axial periodicity of the thread and is the only the lattice parameter that was relaxed. All atomic positions were fully relaxed. A k-spacing of 0.2 $\AA^{-1}$ in the axial direction was used. Thread arching in Figure 7 was computed using at the PBE1PBE-D3BJ/6-31G(d) level in Gaussian 09.

\section{Acknowledgement}

We thank Roberto Cammi for fruitful discussions on the XP-PCM calculations. We acknowledge the support provided by the Center for Nanothread Chemistry (CNC) as funded by the National Science Foundation (CHE-1832471). We acknowledge the computing resource provided by the Extreme Science and Engineering Discovery Environment (XSEDE) Comet cluster at the San Diego Supercomputer Center through allocation CHE180059. 


\section{References}

\footnotetext{
${ }^{1}$ Mehta, G.; Viswanath, M. B.; Kunwar, A. C. Characterization of [n]-Ladderanes of Unprecedented Length: A New Record for Fused Carbocyclic Arrays. J. Org. Chem. 1994, 59 (21), 6131-6132 DOI: 10.1021/jo00100a002.

${ }^{2}$ Scherf, U. Ladder-type materials. J. Mater. Chem. 1999, 9 (9), 1853-1864 DOI: 10.1039/A900447E.

${ }^{3}$ Fitzgibbons, T. C.; Guthrie, M.; Xu, E.-S.; Crespi, V. H.; Davidowski, S. K.; Cody, G. D.; Alem, N.; Badding, J. V. Benzene-derived carbon nanothreads. Nat Mater 2015, 14 (1), 43-47 DOI: 10.1038/nmat4088.088

${ }^{4}$ Li, X.; Baldini, M.; Wang, T.; Chen, B.; Xu, E.-S.; Vermilyea, B.; Crespi, V. H.; Hoffmann, R.; Molaison, J. J.; Tulk, C. A.; et al. Mechanochemical Synthesis of Carbon Nanothread Single Crystals. J. Am. Chem. Soc. 2017, 139 (45), 16343-16349 DOI: 10.1021/jacs.7b09311.

${ }^{5}$ Li, X.; Wang, T.; Duan, P.; Baldini, M.; Huang, H.-T.; Chen, B.; Juhl, S. J.; Koeplinger, D.; Crespi, V. H.; Schmidt-Rohr, K.; et al. Carbon Nitride Nanothread Crystals Derived from Pyridine. J. Am. Chem. Soc. 2018, 140 (15), 4969-4972 DOI: 10.1021/jacs.7b13247.
}

${ }^{6}$ Nobrega, M. M.; Teixeira-Neto, E.; Cairns, A. B.; Temperini, M. L. A.; Bini, R. One-dimensional diamondoid polyaniline-like nanothreads from compressed crystal aniline. Chem. Sci. 2018, 9 (1), 254-260 DOI:

10.1039/C7SC03445H.

${ }^{7}$ Ward, M. D.; Tang, W. S.; Zhu, L.; Popov, D.; Cody, G. D.; Strobel, T. A. Controlled Single-Crystalline Polymerization of C10H8·C10F8 under Pressure. Macromolecules 2019, 52 (20), 7557-7563 DOI: 10.1021/acs.macromol.9b01416.

${ }^{8}$ Biswas, A.; Ward, M. D.; Wang, T.; Zhu, L.; Huang, H.-T.; Badding, J. V.; Crespi, V. H.; Strobel, T. A. Evidence for Orientational Order in Nanothreads Derived from Thiophene. J. Phys. Chem. Lett. 2019, 10 (22), 7164-7171 DOI: $10.1021 /$ acs.jpclett.9b02546.

${ }^{9}$ Huang, H.-T.; Zhu, L.; Ward, M. D.; Wang, T.; Chen, B.; Chaloux, B. L.; Wang, Q.; Biswas, A.; Gray, J. L.; Kuei, B.; et al. Nanoarchitecture through Strained Molecules: Cubane-Derived Scaffolds and the Smallest Carbon Nanothreads. J. Am. Chem. Soc. 2020, 142 (42), 17944-17955 DOI: 10.1021/jacs.9b12352.

${ }^{10}$ Gerthoffer, M. C.; Wu, S.; Chen, B.; Wang, T.; Huss, S.; Oburn, S. M.; Crespi, V. H.; Badding, J. V.; Elacqua, E. "Sacrificial" supramolecular assembly and pressure-induced polymerization: toward sequence-defined functionalized nanothreads. Chem. Sci. 2020, 11 (42), 11419-11424 DOI: 10.1039/D0SC03904G.

${ }^{11}$ Huss, S.; Wu, S.; Chen, B.; Wang, T.; Gerthoffer, M. C.; Ryan, D. J.; Smith, S. E.; Crespi, V. H.; Badding, J. V.; Elacqua, E. Scalable Synthesis of Crystalline One-Dimensional Carbon Nanothreads through Modest-Pressure Polymerization of Furan. ACS Nano 2021, 15 (3), 4134-4143 DOI: 10.1021/acsnano.0c10400.

${ }^{12}$ Chen, B.; Hoffmann, R.; Cammi, R. The Effect of Pressure on Organic Reactions in Fluids-a New Theoretical Perspective. Angew. Chem. Int. Ed 2017, 56 (37), 11126-11142 DOI: 10.1002/anie.201705427.

${ }^{13}$ Chen, B.; Hoffmann, R.; Ashcroft, N. W.; Badding, J.; Xu, E.; Crespi, V. Linearly Polymerized Benzene Arrays As Intermediates, Tracing Pathways to Carbon Nanothreads. J. Am. Chem. Soc. 2015, 137 (45), 14373-14386 DOI: 10.1021/jacs.5b09053.

${ }^{14}$ Xu, E.-S.; Lammert, P. E.; Crespi, V. H. Systematic Enumeration of sp3 Nanothreads. Nano Lett. 2015, 15 (8), 5124-5130 DOI: 10.1021/acs.nanolett.5b01343. 
${ }^{15}$ Chen, B.; Wang, T.; Crespi, V. H.; Li, X.; Badding, J.; Hoffmann, R. All the Ways To Have Substituted Nanothreads. J. Chem. Theory Comput. 2018, 14 (2), 1131-1140 DOI: 10.1021/acs.jctc.7b00911.

${ }^{16}$ Matsuura, B.; Huss, S.; Zheng, Z.; Yuan, S.; Wang, T.; Badding, J. V.; Trauner, D.; Elacqua, E.; van Duin, A. C. T.; Crespi, V. H.; Schmidt-Rohr, K. in preparation.

${ }^{17}$ Vessally, E. Aromatic stability energy studies on five-membered heterocyclic C4H4M (M = O, S, Se, Te, NH, PH, AsH and SbH): DFT calculations. Journal of Structural Chemistry 2009, 49 (6), 979-985 DOI: 10.1007/s10947-008-0169-2.

${ }^{18}$ Najmidin, K.; Kerim, A.; Abdirishit, P.; Kalam, H.; Tawar, T. A comparative study of the aromaticity of pyrrole, furan, thiophene, and their aza-derivatives. Journal of Molecular Modeling 2013, 19 (9), 3529-3535 DOI: 10.1007/s00894-013-1877-x.

${ }^{19}$ Anslyn, E. V.; Dougherty, D. A. Modern Physical Organic Chemistry; University Science Books, 2006.

${ }^{20}$ Bidirectional propagation of chain-growth polycondensation: Its application to poly(ethylene glycol)-aromatic polyamide-poly(ethylene glycol) triblock copolymer with low polydispersity. 2003, 41, 1341-1346 DOI: 10.1002/pola.10662.

${ }^{21}$ Rubin, M. D; Gleiter, R. The Chemistry of Vicinal Polycarbonyl Compounds. Chem. Rev.2000, 100, 1121-1164; Frapper, G.; Cu, C.-X.; Halet, J.-F.; Saillard, J. Y.; Kertesz, M. Can carbon monoxide polymerize? A theoretical investigation of polyketone. Chem. Commun. 1997, 2011-2012. Cui, C.-X.; Kertesz, M. Helical Peierls distortion: Formation of helices of polyketone and polyisocyanide. Chem. Phys. Lett. 1990, 169, 445-449.

${ }^{22}$ Genin, H.; Hoffmann, R. Polythiene, a Novel Hypothetical Carbon-Sulfur Polymer. J. Am. Chem. Soc. 1995, 117 (49), 12328-12335 DOI: 10.1021/ja00154a036.

${ }^{23}$ Regarding the bonding in the two $(\mathrm{CX})_{\mathrm{n}}$ polymers $(\mathrm{X}=\mathrm{O}$ or $\mathrm{S})$, polyketone $(\mathrm{CO})_{\mathrm{n}}$ can be viewed as $\mathrm{C}-\mathrm{C}$ singlebond-connected carbonyls $(\mathrm{C}=\mathrm{O})$; each $\mathrm{O}$ atom has two lone pairs, and there is no covalent bonding between $\mathrm{O}$ atoms. Whereas in polythiene (CS)n, the lone pair electrons are partially transferred into the norminal $\mathrm{C}=\mathrm{S} \pi^{*}$ orbital due to the large repulsion between $\mathrm{S}$ lone pairs, resulting in partial bonding between the $\mathrm{S}$ atoms.

${ }^{24}$ Bondi, A. van der Waals Volumes and Radii. The Journal of Physical Chemistry 1964, 68 (3), 441-451 DOI: 10.1021/j100785a001.

${ }^{25}$ Rahm, M.; Hoffmann, R.; Ashcroft, N. W. Atomic and Ionic Radii of Elements 1-96. Chemistry 2016, 22 (41), 14625-14632 DOI: 10.1002/chem.201602949.

${ }^{26}$ Demingos, P. G.; Balzaretti, N. M.; Muniz, A. R. First-principles study of carbon nanothreads derived from fivemembered heterocyclic rings: thiophene, furan and pyrrole. Phys. Chem. Chem. Phys. 2021, 23 (3), 2055-2062 DOI: $10.1039 / \mathrm{d} 0 \mathrm{cp} 05847 \mathrm{e}$.

${ }^{27}$ Grimme, W.; Gossel, J.; ChemInform, J. L. Diels-Alder Oligomers of Benzene. In Modular Chemistry; Springer, 2012; pp 485-488.

${ }^{28}$ Frisch, M. J.; Trucks, G. W.; Schlegel, H. B.; Scuseria, G. E.; Robb, M. A.; Cheeseman, J. R.; Scalmani, G.; Barone, V.; Mennucci, B.;Petersson, G. A.; Nakatsuji, H.; Caricato, M.; Li, X.; Hratchian, H. P.; Izmaylov, A. F.; Bloino, J.; Zheng, G.; Sonnenberg, J. L.; Hada, M.; Ehara, M.; Toyota, K.; Fukuda, R.; Hasegawa, J.; Ishida, M.; Nakajima, T.; Honda, Y.; Kitao, O.; Nakai, H.; Vreven, T.; Montgomery Jr. J. A.; Peralta, J. E.; Ogliaro, F.; Bearpark, M.; Heyd, J. J.; Brothers, E.; Kudin, K. N.; Staroverov, V. N.; Kobayashi, R.; Normand, J.; Raghavachari, K.; Rendell, A.; Burant, J. C.; Iyengar, S. S.; Tomasi, J.; Cossi, M.; Rega, N.; Millam, N. J.; Klene, M.; Knox, J. E.; Cross, J. B.; Bakken, V.; Adamo, C.; Jaramillo, J.; Gomperts, R.; Stratmann, R. E.; Yazyev, O.; Austin, A. J.; Cammi, R.; Pomelli, C.; Ochterski, J. W.; Martin, R. L.; Morokuma, K.; Zakrzewski, V. G.; Voth, G. A.; Salvador, P.; Dannenberg, J. J.; Dapprich, S.; Daniels, A. D.; Farkas, O.; Foresman, J. B.; Ortiz, J. V.; Cioslowski, J.; Fox, D. J. Gaussian 09, revision D.01, Gaussian, Inc.: Wallingford, CT, 2016. 
${ }^{29}$ Becke, A. D. Density-functional thermochemistry. III. The role of exact exchange. J. Chem. Phys. 1993, 98 (7), 5648-5652 DOI: 10.1063/1.464913.

${ }^{30}$ Self-Consistent Molecular-Orbital Methods. IX. An Extended Gaussian-Type Basis for Molecular-Orbital Studies of Organic Molecules. 1971, 54 (2), 724-728 DOI: 10.1063/1.1674902.

${ }^{31}$ Hariharan, P. C.; Pople, J. A. The influence of polarization functions on molecular orbital hydrogenation energies. Theoret. Chim. Acta 1973, 28 (3), 213-222.

${ }^{32}$ Francl, M. M.; Pietro, W. J.; Hehre, W. J.; Binkley, J. S.; Gordon, M. S.; DeFrees, D. J.; Pople, J. A. Selfconsistent molecular orbital methods. XXIII. A polarization-type basis set for second-row elements. J. Chem. Phys. 1982, 77 (7), 3654-3665 DOI: 10.1063/1.444267.

${ }^{33}$ Guo, Y.; Riplinger, C.; Becker, U.; Liakos, D. G.; Minenkov, Y.; Cavallo, L.; Neese, F. Communication: An improved linear scaling perturbative triples correction for the domain based local pair-natural orbital based singles and doubles coupled cluster method [DLPNO-CCSD(T)]. J. Chem. Phys. 2018, 148 (1), 011101 DOI: $10.1063 / 1.5011798$.

${ }^{34}$ Dunning, T. H., Jr. Gaussian basis sets for use in correlated molecular calculations. I. The atoms boron through neon and hydrogen. J. Chem. Phys. 1989, 90 (2), 1007-1023 DOI: 10.1063/1.456153.

${ }^{35}$ Prascher, B. P.; Woon, D. E.; Peterson, K. A.; Dunning, T. H.; Wilson, A. K. Gaussian basis sets for use in correlated molecular calculations. VII. Valence, core-valence, and scalar relativistic basis sets for $\mathrm{Li}, \mathrm{Be}, \mathrm{Na}$, and Mg. Theoret. Chim. Acta 2011, 128 (1), 69-82.

${ }^{36}$ Weigend, F.; Köhn, A.; Hättig, C. Efficient use of the correlation consistent basis sets in resolution of the identity MP2 calculations. Journal of Chemical Physics 2002, 116 (8), 3175-3183 DOI: 10.1063/1.1445115.

${ }^{37}$ Hättig, C. Optimization of auxiliary basis sets for RI-MP2 and RI-CC2 calculations: Core-valence and quintuple$\zeta$ basis sets for $\mathrm{H}$ to Ar and QZVPP basis sets for Li to Kr. Physical Chemistry Chemical Physics 2005, 7 (1), 59-66 DOI: $10.1039 / \mathrm{B} 415208 \mathrm{E}$.

${ }^{38}$ Neese, F. The ORCA program system. WIREs Comput Mol Sci 2012, 2 (1), $73-78$ DOI: 10.1002/wcms.81.

${ }^{39}$ Neese, F. Software update: the ORCA program system, version 4.0. WIREs Comput Mol Sci 2018, 8 (1), e1327 DOI: $10.1002 / \mathrm{wcms} .1327$.

${ }^{40}$ Cammi, R. A new extension of the polarizable continuum model: Toward a quantum chemical description of chemical reactions at extreme high pressure. J. Comput. Chem. 2015, 36 (30), 2246-2259 DOI: 10.1002/jcc.24206.

${ }^{41}$ Chai, J.-D.; Head-Gordon, M. Long-range corrected hybrid density functionals with damped atom-atom dispersion corrections. Physical Chemistry Chemical Physics 2008, 10 (44), 6615-6620 DOI: 10.1039/b810189b.

${ }^{42}$ Weigend, F.; Ahlrichs, R. Balanced basis sets of split valence, triple zeta valence and quadruple zeta valence quality for $\mathrm{H}$ to Rn: Design and assessment of accuracy. Physical Chemistry Chemical Physics 2005, 7 (18), 3297 3305 DOI: 10.1039/b508541a.

${ }^{43}$ Weigend, F. Accurate Coulomb-fitting basis sets for H to Rn. Physical Chemistry Chemical Physics 2006, 8 (9), 1057-1065 DOI: 10.1039/B515623H.

${ }^{44}$ Purvis, G. D., III; Bartlett, R. J. A full coupled-cluster singles and doubles model: The inclusion of disconnected triples. J. Chem. Phys. 1982, 76 (4), 1910-1918 DOI: 10.1063/1.443164.

${ }^{45}$ Pople, J. A.; Head-Gordon, M.; Raghavachari, K. Quadratic configuration interaction. A general technique for determining electron correlation energies. J. Chem. Phys. 1987, 87 (1), 5968-5975 DOI: 10.1063/1.453520.

${ }^{46}$ Velde, te, G.; Bickelhaupt, F. M.; Baerends, E. J.; Fonseca Guerra, C.; van Gisbergen, S. J. A.; Snijders, J. G.; Ziegler, T. Chemistry with ADF. J. Comput. Chem. 2001, 22 (9), 931-967 DOI: 10.1002/jcc.1056. 
${ }^{47}$ Elstner, M.; Porezag, D.; Jungnickel, G.; Elsner, J.; Haugk, M.; Frauenheim, T.; Suhai, S.; Seifert, G. Selfconsistent-charge density-functional tight-binding method for simulations of complex materials properties. Phys. Rev. B 1998, 58 (11), 7260-7268.

${ }^{48}$ Wahiduzzaman, M.; Oliveira, A. F.; Philipsen, P.; Zhechkov, L.; van Lenthe, E.; Witek, H. A.; Heine, T. DFTB Parameters for the Periodic Table: Part 1, Electronic Structure. J. Chem. Theory Comput. 2013, 9 (9), 4006-4017 DOI: $10.1021 / \mathrm{ct} 4004959$.

${ }^{49}$ Oliveira, A. F.; Philipsen, P.; Heine, T. DFTB Parameters for the Periodic Table, Part 2: Energies and Energy Gradients from Hydrogen to Calcium. J. Chem. Theory Comput. 2015, 11 (11), 5209-5218 DOI: 10.1021/acs.jctc.5b00702.

${ }^{50}$ Kresse, G.; Hafner, J. Ab initio molecular dynamics for liquid metals. Phys. Rev. B 1993, 47 (1), 558-561.

${ }^{51}$ Perdew, J. P.; Burke, K.; Ernzerhof, M. Generalized Gradient Approximation Made Simple. Phys. Rev. Lett. 1996, 77 (18), 3865-3868.

${ }^{52}$ Perdew, J. P.; Burke, K.; Ernzerhof, M. Generalized Gradient Approximation Made Simple [Phys. Rev. Lett. 77, 3865 (1996)]. Phys. Rev. Lett. 1997, 78 (7), 1396-1396 DOI: 10.1103/PhysRevLett.78.1396.

${ }^{53}$ Grimme, S.; Ehrlich, S.; Goerigk, L. Effect of the damping function in dispersion corrected density functional theory. J. Comput. Chem. 2011, 32 (7), 1456-1465 DOI: 10.1002/jcc.21759. 